\title{
EDITORIAL
}

\section{Another brick in the wall: adrenomedullin and prognosis in community-acquired pneumonia}

\author{
Stefan Krüger and Dirk Frechen
}

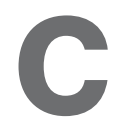

ommunity-acquired pneumonia (CAP) continues to be a major healthcare problem. The associated mortality of $14 \%$ in hospitalised patients is still high [1]. Established clinical scores, such as the Pneumonia Severity Index (PSI) and CRB-65 score (confusion, respiratory frequency $\geqslant 30$ breath$\mathrm{s} \cdot \mathrm{min}^{-1}$, systolic blood pressure $<90 \mathrm{mmHg}$ or diastolic blood pressure $\leqslant 60 \mathrm{mmHg}$, and age $\geqslant 65 \mathrm{yrs}$ ) are used for the determination of mortality risk. Since CAP is an infectious disease, we traditionally use biomarkers of infection for diagnostic and prognostic purposes. Inflammatory markers such as white blood cell (WBC) count and C-reactive protein (CRP) are still widely used in the management of CAP, although it is well known that their value for the diagnosis of clinically relevant bacterial infection and prediction of death and complications in CAP is limited. With respect to better diagnosis and guidance of antibiotic therapy, procalcitonin (PCT) is much better, with a broad spectrum of interventional studies in the field of lower respiratory tract infections [2]. However, PCT is not the ideal biomarker for prognosis in $\mathrm{CAP}$, where cardiovascular biomarkers show better predictive value [3-5].

In the current issue of the European Respiratory Journal, BELLO et al. [6] adds another brick to the multifaceted wall of prognostic assessment in CAP. The authors performed a prospective study in immunocompetent CAP patients and evaluated whether the prognostic role of pro-adrenomedullin (proADM) depends on different aetiologies of CAP. They compared the prognostic value of proADM with that of the clinical severity scores PSI and CURB-65 (confusion, urea $>7 \mathrm{mmol} \cdot \mathrm{L}^{-1}$, respiratory frequency $\geqslant 30$ breaths $\cdot \mathrm{min}^{-1}$, systolic blood pressure $<90 \mathrm{mmHg}$ or diastolic blood pressure $\leqslant 60 \mathrm{mmHg}$, and age $\geqslant 65 \mathrm{yrs}$ ) for the prediction of death at 30, 90 and 180 days, and $1 \mathrm{yr}$. In $68 \%$ of the 228 CAP patients, at least one pathogen was identified. They found that proADM showed a high predictive power with respect to complications and short- and long-term mortality. The addition of proADM to the PSI and CURB-65 score significantly improved the prognostic accuracy. A proADM cut-off of $0.646 \mathrm{ng} \cdot \mathrm{mL}^{-1}$ identified high-risk patients. The predictive power of proADM was not influenced by different aetiologies.

This study provides additional evidence that the primary focus on microbiological aetiology in CAP is misleading. The main focus has to be on severity of CAP, which determines mortality

Medical Clinic I, University Clinic RWTH Aachen, Aachen, Germany.

CORRESPONDENCE: S. Krüger, Medical Clinic I, University Hospital, RWTH University Aachen, Pauwelsstr. 30, D - 52057 Aachen, Germany. E-mail: stkrueger@ukaachen.de and complications. Therefore, it is not surprising that the prognostic value of proADM is independent of microbiological aetiology. No matter what the aetiological agent is, if it results in severe disease, the patient is at risk. This risk has to be identified as soon as possible and ideally at the initial presentation of the patient, within minutes. For this purpose, CAP scores are calculated or biomarkers used because a more rapid initiation of appropriate therapy results in a better outcome for the patients with moderate-to-severe disease.

The results of the present study are comparable to previous studies on the prognostic value of proADM in CAP [3, 7-9]. In the German Competence Network for the Study of Community Acquired Pneumonia (CAPNETZ) substudy, it was shown in 728 patients that proADM was independent of CRB-65 score and added prognostic information for short- and long-term mortality [3]. The main finding of this study was that all cardiovascular biomarkers were good predictors of short- and long-term mortality, superior to the inflammatory markers PCT, CRP and WBC and at least comparable to the clinical CRB65 score. Among the cardiovascular biomarkers, proADM showed the best performance. The results are supported by the biomarker studies from the ProHOSP trial [7]. During a 30day follow-up, 134 serious complications occurred in 925 CAP patients. Both PSI and CURB-65 score overestimated the observed mortality. ProADM alone had stronger discriminatory power compared with the PSI and CURB-65 score for the prediction of serious complications. The addition of proADM to the PSI and CURB-65 score significantly improved the prediction of serious complications. In another study the prognostic value of proADM was comparable to the PSI score and superior to other clinical and laboratory parameters. Again, the combination of proADM and PSI score improved mortality prediction [8]. HUANG et al. [9] found that high proADM levels offer additional risk stratification in high-risk CAP patients only. Altogether, there are now five studies underlining that proADM improves the prognostic value of the established risk scores in CAP.

Why should proADM or other cardiovascular biomarkers be useful for the prediction of survival in patients with CAP? The main question when looking at the mortality of a disease is: what were the reasons for death? Why do patients with CAP die? Short-term mortality is mainly due to sepsis and respiratory failure but also to cardiovascular events. The main cause of longterm mortality is cardiovascular disease. In the setting of CAP, underlying and possibly previously unknown cardiovascular or renal disease may be aggravated due to acute inflammatory 
activation. The elevation of proADM might be attributable to hidden cardiovascular comorbidities or to cardiovascular dysfunction as a consequence of the acute infection of CAP, leaving the patient at increased risk of death. For this reason proADM is useful not only for the prediction of short-term but also for longterm prognosis.

Scores have one problem, as can be seen again in this study: they are composed of several parameters, which make the calculation of the scores time consuming. The CRB-65 and PSI score are validated for CAP. Although the PSI score includes 20 single parameters with the intention to compose a complex system of different pathophysiological aspects of a disease, the score is not superior to proADM as a single biomarker to predict mortality. There is only one sensible explanation for why a biomarker such as proADM might be better compared with a complex score: the biomarker is not an indicator for one exclusive disease or single physiological disturbance. A relevant biomarker for prognosis, such as proADM, reflects not a single but multiple pathophysiological aspects of a disease. In the case of proADM these aspects are not only cardiovascular activity but also antiinflammatory and antibacterial functions.

What could biomarkers in CAP be used for in the future? There is overwhelming evidence that PCT can be used for the better diagnosis of CAP and for the guidance of antibiotic therapy, a very important issue in order to reduce selection pressure and multi-drug resistance [2]. ProADM could be used for the identification of patients with a high risk of mortality. In these patients, increased attention to possible cardiovascular disease and closer medical follow-up may be indicated and could result in a better outcome. Whether the better predictive potential of proADM translates into clinically relevant prediction tools of mortality from CAP remains to be determined. A combination of a clinical score, such as CURB-65 or PSI, with proADM for risk assessment is an attractive approach currently under validation in independent future cohorts $[10,11]$. Thus, we have to await the results of future studies in CAP, such as OPTIMA II, applying proADM for the choice of treatment site. The future of biomarkers and risk prediction in CAP remains exciting and has just begun.

\section{STATEMENT OF INTEREST}

A statement of interest for S. Krüger can be found at www.erj. ersjournals.com/site/misc/statements.xhtml

\section{REFERENCES}

1 Ewig S, Birkner N, Strauss R, et al. New perspectives on community-acquired pneumonia in 388,406 patients. Results from a nationwide mandatory performance measurement programme in healthcare quality. Thorax 2009; 64: 1062-1069.

2 Schuetz P, Albrich W, Christ-Crain M, et al. Procalcitonin for guidance of antibiotic therapy. Expert Rev Anti Infect Ther 2010; 8: 575-587.

3 Krüger S, Ewig S, Giersdorf S, et al. Cardiovascular and inflammatory biomarkers to predict short- and long-term survival in community-acquired pneumonia. Results from the German competence network CAPNETZ. Am J Respir Crit Care Med 2010; 182: $1426-1434$

4 Krüger S, Ewig S, Marre R, et al. Procalcitonin predicts patients at low risk of death from community-acquired pneumonia across all CRB-65 classes. Eur Respir J 2008; 31: 349-355.

5 Krüger S, Ewig S, Kunde J, et al. Pro-atrial natriuretic peptide and pro-vasopressin to predict short- and long-term survival in community-acquired pneumonia. Results from the German competence network CAPNETZ. Thorax 2010; 65: 208-214.

6 Bello S, Lasierra AB, Mincholé E, et al. Prognostic power of proadrenomedullin in community-acquired pneumonia is independent of aetiology. Eur Respir J 2012; 39: 1144-1155.

7 Schuetz P, Wolbers M, Christ-Crain M, et al. Prohormones for prediction of adverse medical outcome in community-acquired pneumonia and lower respiratory tract infections. Crit Care 2010; 14: R106.

8 Christ-Crain M, Morgenthaler NG, Stolz D, et al. Pro-adrenomedullin to predict severity and outcome in community-acquired pneumonia [ISRCTN04176397]. Crit Care 2006; 10: R96.

9 Huang DT, Angus DC, Kellum JA, et al. Midregional proadrenomedullin as a prognostic tool in community-acquired pneumonia Chest 2009; 136: 823-831.

10 Albrich WC, Dusemund F, Rüegger K, et al. Enhancement of CURB65 score with proadrenomedullin (CURB65-A) for outcome prediction in lower respiratory tract infections: derivation of a clinical algorithm. BMC Infect Dis 2011; 11: 112.

11 Albrich WC, Rüegger K, Dusemund F, et al. Optimised patient transfer using an innovative multidisciplinary assessment in Kanton Aargau (OPTIMA I): an observational survey in lower respiratory tract infections. Swiss Med Wkly 2011; 141: w13237. 